\title{
Stabilization of the Trial Method for the Bernoulli Problem in Case of Prescribed Dirichlet Data
}

\author{
Helmut Harbrecht, Giannoula Mitrou
}

Institute of Mathematics

Preprint No. 2013-29

University of Basel

December, 2013

Rheinsprung 21

CH - 4051 Basel

Switzerland

$\underline{\text { www.math.unibas.ch }}$ 


\title{
Stabilization of the trial method for the Bernoulli problem in case of prescribed Dirichlet data
}

\author{
Helmut Harbrecht $^{\mathrm{a} *}$, Giannoula Mitrou ${ }^{\mathrm{a}}$
}

We apply the trial method for the solution of Bernoulli's free boundary problem when the Dirichlet boundary condition is imposed for the solution of the underlying Laplace equation and the free boundary is updated according to the Neumann boundary condition. The Dirichlet boundary value problem for the Laplacian is solved by an exponentially convergent boundary element method. The update rule for the free boundary is derived from the linearization of the Neumann data around the actual free boundary. With the help of shape sensitivity analysis and Banach's fixed-point theorem, we shed light on the convergence of the respective trial method. Especially, we derive a stabilized version of this trial method. Numerical examples validate the theoretical findings. Copyright (c) 2009 John Wiley \& Sons, Ltd.

Keywords: free boundary problems; boundary element method; trial method; fixed-point method

\section{Introduction}

\subsection{Motivation and background}

Free boundary problems are boundary value problems which include a partial differential equation in the domain and boundary conditions on the boundary of the domain, a part of which is unknown, the so-called free boundary. On the free boundary, there are given the complete Cauchy data which serve different purposes. The Dirichlet boundary condition is to solve the differential equation and the Neumann boundary condition is to find the geometry of the free boundary, or vice versa. This means for a numerical method that the free boundary can be updated either according to the Dirichlet boundary condition at the free boundary or according to the Neumann boundary condition. In general, this choice depends on the physical properties of the free boundary problem under consideration. Among the existing methods for solving free boundary problems, such as the level set method [5, 14] or the shape optimization method [8, 11, 12, 23], we investigate here the trial method [6, 9, 10, 23, 24].

Unlike the usual technique of updating the free boundary according to the Dirichlet boundary condition, we derive update rules by updating according to the Neumann boundary condition. Some theoretical results concerning the convergence of the respective trial method can be found in [1]. There is nevertheless, to the best of our knowledge, no article with numerical results on this trial method apart from the case of axially symmetric domains. For instance, in [20], there has been shown that updating the free boundary according to the Neumann boundary condition makes the trial method easier under certain circumstances.

\subsection{Bernoulli's free boundary problem}

Bernoulli's free boundary problem can be viewed as the prototype of a large class of stationary free boundary problems involved in many applications such as fluid dynamics, optimal design, electromagnetics, and various other engineering fields. We refer to $[3,4,22]$ for a review of theoretical results concerning the existence and uniqueness of solutions to Bernoulli's free boundary problem. Results on the geometric form of the free boundary can be found in [2] and the references therein.

Let $T \subset \mathbb{R}^{2}$ denote a bounded domain with free boundary $\partial T=\Gamma$. Inside the domain $T$, we assume the existence of a simply connected subdomain $S \subset T$ with fixed boundary $\partial S=\Sigma$. The resulting annular domain $T \backslash \bar{S}$ is denoted by $\Omega$ and displayed in Figure 1.1. Bernoulli's free boundary problem consists now in seeking the domain $\Omega$ and the function $u$ which both satisfy the 


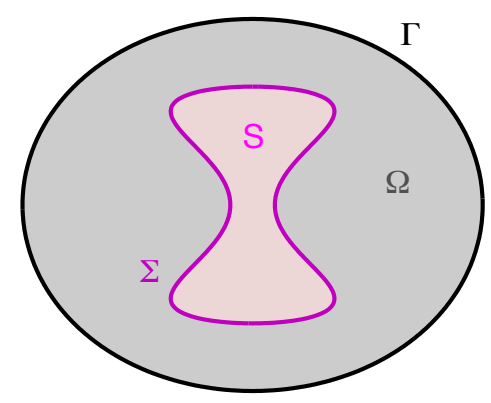

Figure 1.1. The domain $\Omega$ and its boundaries $\Gamma$ and $\Sigma$

following overdetermined boundary value problem:

$$
\begin{array}{rlrl}
\Delta u & =0 & & \text { in } \Omega \\
u=1 & & \text { on } \Sigma \\
u & =0 & & \text { on } \Gamma \\
-\frac{\partial u}{\partial \mathbf{n}} & =\lambda & & \text { on } \Gamma .
\end{array}
$$

Here, the vector $\mathbf{n}$ denotes the outward normal vector at the boundary $\Gamma$ and $\partial u / \partial \mathbf{n}$ denotes the derivative of $u$ in the normal direction. Furthermore, the Neumann data $\lambda$ are supposed to be positive and constant such that the solution $u$ to (1.1) is well defined and positive in $\Omega$.

For the solution of the boundary value problem and for the subsequent analysis, we make the following assumption:

Assumption 1.1 We assume that the domain $T$ is starlike. The free boundary $\Gamma$ can thus be parametrized in polar coordinates as

$$
\boldsymbol{\gamma}:[0,2 \pi] \rightarrow \Gamma, \quad s \mapsto \boldsymbol{\gamma}(s)=r(s) \mathbf{e}_{r}(s),
$$

where $\mathbf{e}_{r}(s)=(\cos (s), \sin (s))^{T}$ stands for the unit radial vector. In particular, the radial function $r(s)$ is supposed to be a positive function in $C_{\text {per }}^{2}([0,2 \pi])$, where

$$
C_{\mathrm{per}}^{2}([0,2 \pi])=\left\{r \in C^{2}([0,2 \pi]): r^{(i)}(0)=r^{(i)}(2 \pi), \quad i=0,1,2\right\},
$$

such that $\operatorname{dist}(\Sigma, \Gamma)>0$.

\subsection{Trial method}

A prominent method to solve the free boundary value problem (1.1) under consideration is the trial method. It is a fixed-point type method. Here, we fix the pure Dirichlet problem as state equation and aim at updating the free boundary such that the desired Neumann boundary condition is fulfilled at the new boundary. The basic idea of this iterative scheme is described in the following algorithm:

\section{Algorithm 1.2 : Trial method}

1. Choose an initial guess $\Gamma_{0}$ of the free boundary.

2. (a) Compute the Neumann data of $u_{k}$ at the boundary $\Gamma_{k}$ by applying the boundary element method to the boundary value problem

$$
\begin{array}{rlr}
\Delta u_{k}=0 & & \text { in } \Omega_{k} \\
u_{k}=1 & & \text { on } \Sigma \\
u_{k}=0 & & \text { on } \Gamma_{k} .
\end{array}
$$

(b) Update the free boundary according to the update rule

$$
\boldsymbol{\gamma}_{k+1}=\boldsymbol{\gamma}_{k}+\beta \delta r_{k} \mathbf{e}_{r}
$$

The update $\delta r_{k} \in C_{\text {per }}^{2}([0,2 \pi])$ is determined by Taylor's expansion of the Neumann data, such that the Neumann boundary condition is approximately satisfied at the new boundary $\Gamma_{k+1}$. The parameter $\beta$ stands for a correction factor.

3. Iterate step 2 until the process becomes stationary up to a specified precision. 
The use of a first order Taylor expansion when the free boundary is updated according to the Dirichlet boundary condition at the boundary $\Gamma_{k}$ has been proposed for example in $[9,24]$.

\subsection{Organization of the article}

The remainder of this article is organized as follows. In Section 2, after a brief review of results from shape sensitivity analysis, we compute the Neumann data's first order Taylor expansion around the actual free boundary. This yields a first update rule for the free boundary. In Section 3, the numerical realization of the free boundary problem is introduced. First, the free boundary is discretized in Subsection 3.1. Then, in Subsection 3.2, we present the boundary element method to determine the Neumann data of the function $u$. Some numerical tests for the update rule (1.3) are performed in Subsection 3.3. They show numerical difficulties, especially if the free boundary is not convex. Section 4 is thus dedicated to the convergence analysis of the trial method, due to which we propose to modify the update rule such that the convergence is improved. The feasibility of the resulting trial method is shown by numerical results in Subsection 4.4. Finally, in Section 5, the article's conclusion is drawn.

\section{Derivation of the update rule}

\subsection{Background in shape sensitivity analysis}

We shall give a brief background in shape sensitivity analysis, necessary for our further computations. For all the details, especially the proof of Lemma 2.4, we address the reader to $[7,15,16,19]$.

Let $\mathbf{V}: \Omega \rightarrow \mathbb{R}^{2}$ be a sufficiently smooth perturbation field which does not change the interior boundary $\Sigma$, i.e., $\left.\mathbf{V}\right|_{\Sigma}=\mathbf{0}$. Then, given a small parameter $\varepsilon>0$, the perturbed domain $\Omega_{\varepsilon}=\Omega_{\varepsilon}[\mathbf{V}]$ is defined via

$$
\Omega_{\varepsilon}:=\{(\mathbf{I}+\varepsilon \mathbf{V})(\mathbf{x}): \mathbf{x} \in \Omega\} .
$$

Consequently, the associated perturbation of the outer boundary $\Gamma$ is

$$
\Gamma_{\varepsilon}:=\{(\mathbf{I}+\varepsilon \mathbf{V})(\mathbf{x}): \mathbf{x} \in \Gamma\} .
$$

On the domains $\Omega$ and $\Omega_{\varepsilon}$ with interior boundary $\Sigma$ and outer boundaries $\Gamma$ and $\Gamma_{\varepsilon}$, respectively, the functions $u$ and $u_{\varepsilon}$ are defined as the solution to the boundary value problems

$$
\begin{aligned}
\Delta u=0 & \text { in } \Omega, & \Delta u_{\varepsilon}=0 & \text { in } \Omega_{\varepsilon}, \\
u=1 & \text { on } \Sigma, & u_{\varepsilon}=1 & \text { on } \Sigma, \\
u=0 & \text { on } \Gamma, & u_{\varepsilon}=0 & \text { on } \Gamma_{\varepsilon} .
\end{aligned}
$$

The relation between $u$ and $u_{\varepsilon}$ for small values of $\varepsilon$ is subject of the shape sensitivity analysis. As an important concept, the material derivative $\dot{u}$ is introduced. It is computed by pulling back $u_{\varepsilon}$ to the unperturbed domain $\Omega$, i.e., by differentiating $u^{\varepsilon}(\mathbf{x}):=\left(u_{\varepsilon} \circ(\mathbf{I}+\varepsilon \mathbf{V})\right)(\mathbf{x})=u_{\varepsilon}\left(\mathbf{x}_{\varepsilon}\right)$.

Definition 2.1 The material derivative of $u$ in the direction $\mathbf{V}$ is defined as the limit

$$
\dot{u}[\mathbf{V}](\mathbf{x}):=\left.\frac{\mathrm{d} u^{\varepsilon}[\mathbf{V}](\mathbf{x})}{\mathrm{d} \varepsilon}\right|_{\varepsilon=0}=\lim _{\varepsilon \rightarrow 0} \frac{u^{\varepsilon}[\mathbf{V}](\mathbf{x})-u(\mathbf{x})}{\varepsilon}, \quad \mathbf{x} \in \Omega .
$$

In contrast, the direct differentiation of $u_{\varepsilon}(\mathbf{x})$ leads to the local shape derivative.

Definition 2.2 The local shape derivative of $\mathrm{u}$ in the direction $\mathbf{V}$ is given by

$$
\delta u[\mathbf{V}](\mathbf{x}):=\left.\frac{\mathrm{d} u_{\varepsilon}[\mathbf{V}](\mathbf{x})}{\mathrm{d} \varepsilon}\right|_{\varepsilon=0}=\lim _{\varepsilon \rightarrow 0} \frac{u_{\varepsilon}[\mathbf{V}](\mathbf{x})-u(\mathbf{x})}{\varepsilon}, \quad \mathbf{x} \in \Omega .
$$

The relation between the material and the local shape derivative is expressed in the following remark.

Remark 2.3 The chain rule combines the material and the local shape derivative by the relation

$$
\dot{u}[\mathbf{V}]=\delta u[\mathbf{V}]+\langle\nabla u, \mathbf{V}\rangle .
$$

The local shape derivative of $u$ from the boundary value problem (2.2) reads as follows.

Lemma 2.4 The local shape derivative $\delta u$ under the perturbation $\mathbf{V}$ is given as the solution of the boundary value problem

$$
\begin{aligned}
\Delta \delta u & =0 & & \text { in } \Omega \\
\delta u & =0 & & \text { on } \Sigma \\
\delta u & =-\frac{\partial u}{\partial \mathbf{n}}\langle\mathbf{V}, \mathbf{n}\rangle & & \text { on } \Gamma .
\end{aligned}
$$


We finally like to mention at the end of this subsection that a smooth boundary variation $\mathbf{V}: \Gamma \rightarrow \mathbb{R}^{2}$ which acts on the free boundary $\Gamma$ can always smoothly be extended to a domain perturbation $\widetilde{\mathbf{V}}: \Omega \rightarrow \mathbb{R}^{2}$ such that $\left.\widetilde{\mathbf{V}}\right|_{\Gamma}=\mathbf{V}$. This is important for our subsequent analysis since each iteration of the trial method imposes a boundary variation in the direction $\mathbf{V}=\delta r_{k} \mathbf{e}_{r}$.

\subsection{Taylor's expansion}

As step $2 \mathrm{~b}$ of the Algorithm 1.2 indicates, the trial method requires an update rule for the free boundary, which moves the old trial free boundary in the radial direction. Suppose that the actual boundary is $\Gamma_{k}$. Then, the corresponding state $u_{k}$ satisfies the boundary value problem (1.2). The update function $\delta r_{k} \in C_{\text {per }}^{2}([0,2 \pi])$ is found by the requirement that the Neumann boundary condition should be satisfied at the new boundary $\Gamma_{k+1}$, i.e.,

$$
-\frac{\partial u_{k}}{\partial \mathbf{n}} \circ \boldsymbol{\gamma}_{k+1} \stackrel{!}{=} \lambda \quad \text { on }[0,2 \pi] \text {. }
$$

The first order Taylor expansion of the Neumann data in combination with equation (2.5) leads to the update equation

$$
\frac{\partial u_{k}}{\partial \mathbf{n}} \circ \boldsymbol{\gamma}_{k+1} \approx \frac{\partial u_{k}}{\partial \mathbf{n}} \circ \boldsymbol{\gamma}_{k}+\frac{\partial}{\partial\left(\delta r_{k} \mathbf{e}_{r}\right)}\left(\frac{\partial u_{k}}{\partial \mathbf{n}} \circ \boldsymbol{\gamma}_{k}\right)
$$

for the unknown update function $\delta r_{k}$. The Neumann data of the function $u_{k}$ are defined as the inner product of the gradient of the function $u_{k}$ and the normal vector at the boundary $\Gamma_{k}$, i.e.

$$
\frac{\partial u_{k}}{\partial \mathbf{n}} \circ \boldsymbol{\gamma}_{k}=\left\langle\nabla u_{k} \circ \boldsymbol{\gamma}_{k}, \mathbf{n}\right\rangle \text {. }
$$

Thus, by applying the product rule, the directional derivative of the Neumann data of $u_{k}$ is given by

$$
\frac{\partial}{\partial\left(\delta r_{k} \mathbf{e}_{r}\right)}\left(\frac{\partial u_{k}}{\partial \mathbf{n}} \circ \boldsymbol{\gamma}_{k}\right)=\delta r_{k}\left\langle\left(\nabla^{2} u_{k} \circ \boldsymbol{\gamma}_{k}\right) \cdot \mathbf{n}, \mathbf{e}_{r}\right\rangle+\left\langle\nabla u_{k} \circ \boldsymbol{\gamma}_{k}, \frac{\partial \mathbf{n}}{\partial\left(\delta r_{k} \mathbf{e}_{r}\right)}\right\rangle
$$

The right hand side of equation (2.7) contains not only the directional derivative of the Neumann data $\partial^{2} u_{k} /\left(\partial \mathbf{n} \partial \mathbf{e}_{r}\right)$ but also the directional derivative of the normal vector. For the computation of this term, we present Lemma 2.5 where we remind that $\delta r_{k}$ is a scalar function in $C_{\text {per }}^{2}([0,2 \pi])$.

Lemma 2.5 Let $\mathbf{V}=\delta r_{k} \mathbf{e}_{r}$ be the direction of the update of the boundary $\Gamma_{k}$. Then, the directional derivative of the unit normal vector in this direction is given by

$$
\frac{\partial \mathbf{n}}{\partial \mathbf{V}}=\delta r_{k} \frac{\left\langle\mathbf{e}_{r}, \mathbf{t}\right\rangle}{\left\|\boldsymbol{\gamma}_{k}^{\prime}\right\|} \mathbf{t}-\delta r_{k}^{\prime} \frac{\left\langle\mathbf{e}_{r}, \mathbf{n}\right\rangle}{\left\|\boldsymbol{\gamma}_{k}^{\prime}\right\|} \mathbf{t}
$$

where $\mathbf{t}$ indicates the unit tangent vector.

Proof. According to $[7,19]$, the material derivative of the normal vector in the direction of an arbitrary vector field $\mathbf{V}$ is given by

$$
\dot{\mathbf{n}}[\mathbf{V}]=\langle\nabla \mathbf{V} \cdot \mathbf{n}, \mathbf{n}\rangle \mathbf{n}-\nabla \mathbf{V} \cdot \mathbf{n} .
$$

The definition of the normal vector does not depend on the actual domain which means that its local shape derivative vanishes. Therefore, due to Remark 2.3, the directional derivative of the normal vector coincides with its material derivative (2.9).

For the subsequent computations, we require an extension $\widetilde{\delta} r_{k}$ of $\delta r_{k}$ into the neighbourhood of $\Gamma_{k}$. Given a point $\underset{\widetilde{v}}{\mathbf{x}}=\boldsymbol{\gamma}_{k}(s)+\operatorname{tn}(s) \subset \mathbb{R}^{2}$ with $t$ being sufficiently small, we thus set $\widetilde{\delta} r_{k}(\mathbf{x})=\delta r_{k}(s)$. Hence, the gradient of the vector field $\widetilde{\mathbf{v}}=\widetilde{\delta} r_{k} \mathbf{e}_{r}$ reads as

and equation (2.9) becomes

$$
\nabla \widetilde{\mathbf{V}}=\widetilde{\delta} r_{k} \nabla \mathbf{e}_{r}+\nabla \widetilde{\delta} r_{k} \mathbf{e}_{r}^{T}
$$

$$
\begin{aligned}
\frac{\partial \mathbf{n}}{\partial\left(\delta r_{k} \mathbf{e}_{r}\right)} & =\widetilde{\delta r_{k}}\left[\left\langle\nabla \mathbf{e}_{r} \cdot \mathbf{n}, \mathbf{n}\right\rangle \mathbf{n}-\nabla \mathbf{e}_{r} \cdot \mathbf{n}\right]+\left\langle\nabla \widetilde{\delta}_{k} \mathbf{e}_{r}^{T} \cdot \mathbf{n}, \mathbf{n}\right\rangle \mathbf{n}-\nabla \widetilde{\delta r_{k}} \mathbf{e}_{r}^{T} \cdot \mathbf{n} \\
& =-\widetilde{\delta r_{k}}\left\langle\nabla \mathbf{e}_{r} \cdot \mathbf{n}, \mathbf{t}\right\rangle \mathbf{t}-\left\langle\nabla \widetilde{\delta r_{k}} \mathbf{e}_{r}^{T} \cdot \mathbf{n}, \mathbf{t}\right\rangle \mathbf{t} .
\end{aligned}
$$

We compute the terms included in (2.10) as follows. The gradient of the unit radial vector $\mathbf{e}_{r}=\boldsymbol{\gamma}_{k} /\left\|\boldsymbol{\gamma}_{k}\right\|$ is given in polar coordinates ${ }^{\dagger}$ by

$$
\nabla \mathbf{e}_{r}(s)=\frac{1}{\left\|\boldsymbol{\gamma}_{k}(s)\right\|}\left[\begin{array}{r}
-\sin (s) \\
\cos (s)
\end{array}\right] \frac{\partial \mathbf{e}_{r}(s)}{\partial s}=\frac{\mathbf{e}_{r}^{\perp}(s)\left(\mathbf{e}_{r}^{\perp}(s)\right)^{T}}{\left\|\boldsymbol{\gamma}_{k}(s)\right\|}, \quad \text { where } \mathbf{e}_{r}^{\perp}(s)=\left[\begin{array}{r}
-\sin (s) \\
\cos (s)
\end{array}\right]
$$

$\dagger$ The gradient of a function $f(r, \phi)$ given in polar coordinates is computed as

$$
\nabla f(r, \phi)=\frac{1}{r}\left[\begin{array}{rr}
r \cos \phi & -\sin \phi \\
r \sin \phi & \cos \phi
\end{array}\right]\left[\begin{array}{l}
\frac{\partial f}{\partial r} \\
\frac{\partial f}{\partial \phi}
\end{array}\right] .
$$


By use of this relation, the first term of the right hand side of (2.10) is calculated as

$$
\left\langle\nabla \mathbf{e}_{r} \cdot \mathbf{n}, \mathbf{t}\right\rangle \mathbf{t}=\left\langle\frac{\mathbf{e}_{r}^{\perp}\left(\mathbf{e}_{r}^{\perp}\right)^{T}}{\left\|\boldsymbol{\gamma}_{k}\right\|} \cdot \mathbf{n}, \mathbf{t}\right\rangle \mathbf{t}=\frac{\mathbf{t}^{T} \mathbf{e}_{r}^{\perp}\left(\mathbf{e}_{r}^{\perp}\right)^{T} \mathbf{n}}{\left\|\boldsymbol{\gamma}_{k}\right\|} \mathbf{t}=\frac{\left\langle\mathbf{e}_{r}^{\perp}, \mathbf{t}\right\rangle\left\langle\mathbf{e}_{r}^{\perp}, \mathbf{n}\right\rangle}{\left\|\boldsymbol{\gamma}_{k}\right\|} \mathbf{t}
$$

Exploiting the identities

$$
\left\langle\mathbf{e}_{r}^{\perp}, \mathbf{t}\right\rangle=-\left\langle\mathbf{e}_{r}, \mathbf{n}\right\rangle, \quad\left\langle\mathbf{e}_{r}^{\perp}, \mathbf{n}\right\rangle=\left\langle\mathbf{e}_{r}, \mathbf{t}\right\rangle \quad \text { and } \quad \frac{\left\langle\mathbf{e}_{r}, \mathbf{n}\right\rangle}{\left\|\boldsymbol{\gamma}_{k}\right\|}=\frac{1}{\left\|\boldsymbol{\gamma}_{k}^{\prime}\right\|},
$$

(2.11) can be further simplified in accordance with

$$
\left\langle\nabla \mathbf{e}_{r} \cdot \mathbf{n}, \mathbf{t}\right\rangle \mathbf{t}=-\frac{\left\langle\mathbf{e}_{r}, \mathbf{n}\right\rangle\left\langle\mathbf{e}_{r}, \mathbf{t}\right\rangle}{\left\|\boldsymbol{\gamma}_{k}\right\|} \mathbf{t}=-\frac{\left\langle\mathbf{e}_{r}, \mathbf{t}\right\rangle}{\left\|\boldsymbol{\gamma}_{k}^{\prime}\right\|} \mathbf{t}
$$

Employing again polar coordinates, the gradient of $\widetilde{\delta} r_{k}$ is seen to be

$$
\nabla \widetilde{\delta} r_{k}\left(\boldsymbol{\gamma}_{k}(s)+\operatorname{tn}(s)\right)=\frac{1}{\left\|\boldsymbol{\gamma}_{k}(s)\right\|}\left[\begin{array}{r}
-\sin (s) \\
\cos (s)
\end{array}\right] \delta r_{k}(s)^{\prime}=-\frac{\mathbf{e}_{r}^{\perp}(s)}{\left\|\boldsymbol{\gamma}_{k}(s)\right\|} \delta r_{k}(s)^{\prime} .
$$

Consequently, the second term of the right hand side of (2.10) transforms to

$$
\left\langle\nabla \widetilde{\delta} r_{k} \mathbf{e}_{r}^{T} \cdot \mathbf{n}, \mathbf{t}\right\rangle \mathbf{t}=-\frac{1}{\left\|\boldsymbol{\gamma}_{k}\right\|} \delta r_{k}^{\prime}\left\langle\mathbf{e}_{r}^{\perp}, \mathbf{t}\right\rangle\left\langle\mathbf{e}_{r}, \mathbf{n}\right\rangle \mathbf{t}=\delta r_{k}^{\prime} \frac{\left\langle\mathbf{e}_{r}, \mathbf{n}\right\rangle}{\left\|\boldsymbol{\gamma}_{k}^{\prime}\right\|} \mathbf{t} .
$$

The validity of (2.8) follows finally from plugging (2.12) and (2.13) into (2.10).

We proceed with the computation of the derivative of the Neumann data of $u_{k}$ in the direction $\mathbf{V}$.

Lemma 2.6 The derivative of the Neumann data of the function $u_{k}$, which satisfies the boundary value problem (1.2), in the direction $\mathbf{V}=\delta r_{k} \mathbf{e}_{r}$ is given by

$$
\frac{\partial}{\partial\left(\delta r_{k} \mathbf{e}_{r}\right)}\left(\frac{\partial u_{k}}{\partial \mathbf{n}} \circ \boldsymbol{\gamma}_{k}\right)=\left(\left(\frac{\partial^{2} u_{k}}{\partial \mathbf{n}^{2}} \circ \boldsymbol{\gamma}_{k}\right)\left\langle\mathbf{e}_{r}, \mathbf{n}\right\rangle+\left(\frac{\partial^{2} u_{k}}{\partial \mathbf{n} \partial \mathbf{t}} \circ \boldsymbol{\gamma}_{k}\right)\left\langle\mathbf{e}_{r}, \mathbf{t}\right\rangle\right) \delta r_{k} .
$$

Proof. We return to equation (2.7) and notice that the first term of the right-hand side of equation (2.7) is found by decomposing the second order directional derivative of $u_{k}$ into its normal and tangential components as follows:

$$
\begin{aligned}
\left\langle\left(\nabla^{2} u_{k} \circ \boldsymbol{\gamma}_{k}\right) \cdot \mathbf{n}, \mathbf{e}_{r}\right\rangle & =\left\langle\left(\nabla^{2} u_{k} \circ \boldsymbol{\gamma}_{k}\right) \cdot \mathbf{n}, \mathbf{n}\right\rangle\left\langle\mathbf{e}_{r}, \mathbf{n}\right\rangle+\left\langle\left(\nabla^{2} u_{k} \circ \boldsymbol{\gamma}_{k}\right) \cdot \mathbf{n}, \mathbf{t}\right\rangle\left\langle\mathbf{e}_{r}, \mathbf{t}\right\rangle \\
& =\left(\frac{\partial^{2} u_{k}}{\partial \mathbf{n}^{2}} \circ \boldsymbol{\gamma}_{k}\right)\left\langle\mathbf{e}_{r}, \mathbf{n}\right\rangle+\left(\frac{\partial^{2} u_{k}}{\partial \mathbf{n} \partial \mathbf{t}} \circ \boldsymbol{\gamma}_{k}\right)\left\langle\mathbf{e}_{r}, \mathbf{t}\right\rangle .
\end{aligned}
$$

By inserting (2.8) and (2.15) into (2.7), we arrive at the directional derivative of the Neumann data at the boundary $\Gamma_{k}$ :

$$
\frac{\partial}{\partial\left(\delta r_{k} \mathbf{e}_{r}\right)}\left(\frac{\partial u_{k}}{\partial \mathbf{n}} \circ \boldsymbol{\gamma}_{k}\right)=\left[\left(\frac{\partial^{2} u_{k}}{\partial \mathbf{n}^{2}} \circ \boldsymbol{\gamma}_{k}\right)\left\langle\mathbf{e}_{r}, \mathbf{n}\right\rangle+\left(\frac{\partial^{2} u_{k}}{\partial \mathbf{n} \partial \mathbf{t}} \circ \boldsymbol{\gamma}_{k}\right)\left\langle\mathbf{e}_{r}, \mathbf{t}\right\rangle+\left(\frac{\partial u_{k}}{\partial \mathbf{t}} \circ \boldsymbol{\gamma}_{k}\right) \frac{\left\langle\mathbf{e}_{r}, \mathbf{t}\right\rangle}{\left\|\boldsymbol{\gamma}_{k}^{\prime}\right\|}\right] \delta r_{k}-\left(\frac{\partial u_{k}}{\partial \mathbf{t}} \circ \boldsymbol{\gamma}_{k}\right) \frac{\left\langle\mathbf{e}_{r}, \mathbf{n}\right\rangle}{\left\|\boldsymbol{\gamma}_{k}^{\prime}\right\|} \delta r_{k}^{\prime}
$$

Finally, due to the Dirichlet boundary condition $u_{k}=0$ at $\Gamma_{k}$, the tangential derivative of $u_{k}$ vanishes and (2.14) follows.

The directional derivative of the Neumann data of the function $u_{k}$, as it is given in (2.16), coincides with the derivative which has been proven in [18, Theorem 3.11] in the context of inverse scattering problems. However, by using results from shape sensitivity analysis, we were able to obtain this relation by a much simpler proof.

\subsection{Update equation}

With the help of (2.14), we are able to formulate the update equation for the unknown function $\delta r_{k}$ in Lemma 2.7.

Lemma 2.7 The update equation, which is obtained by combining (2.5) and (2.6), reads as

$$
\lambda=\left[\kappa\left(\frac{\partial u_{k}}{\partial \mathbf{n}} \circ \boldsymbol{\gamma}_{k}\right)\left\langle\mathbf{e}_{r}, \mathbf{n}\right\rangle-\frac{\partial}{\partial \mathbf{t}}\left(\frac{\partial u_{k}}{\partial \mathbf{n}} \circ \boldsymbol{\gamma}_{k}\right)\left\langle\mathbf{e}_{r}, \mathbf{t}\right\rangle\right] \delta r_{k}-\frac{\partial u_{k}}{\partial \mathbf{n}} \circ \boldsymbol{\gamma}_{k},
$$

where $\kappa$ denotes the curvature $\kappa=-\left\langle\boldsymbol{\gamma}_{k}^{\prime \prime}, \mathbf{n}\right\rangle /\left\|\boldsymbol{\gamma}_{k}^{\prime}\right\|^{2}$. 
Proof. The combination of the Taylor expansion (2.6) with the requirement (2.5) at the next boundary $\Gamma_{k+1}$ induces the update equation

$$
-\lambda=\frac{\partial u_{k}}{\partial \mathbf{n}} \circ \boldsymbol{\gamma}_{k}+\frac{\partial}{\partial\left(\delta r_{k} \mathbf{e}_{r}\right)}\left(\frac{\partial u_{k}}{\partial \mathbf{n}} \circ \boldsymbol{\gamma}_{k}\right) .
$$

Due to (2.14), this equation can be transformed to

$$
-\lambda=\frac{\partial u_{k}}{\partial \mathbf{n}} \circ \boldsymbol{\gamma}_{k}+\left[\left(\frac{\partial^{2} u_{k}}{\partial \mathbf{n}^{2}} \circ \boldsymbol{\gamma}_{k}\right)\left\langle\mathbf{e}_{r}, \mathbf{n}\right\rangle+\left(\frac{\partial^{2} u_{k}}{\partial \mathbf{n} \partial \mathbf{t}} \circ \boldsymbol{\gamma}_{k}\right)\left\langle\mathbf{e}_{r}, \mathbf{t}\right\rangle\right] \delta r_{k} .
$$

We compute the second order directional derivative $\partial^{2} u_{k} /(\partial \mathbf{n} \partial \mathbf{t})$ by differentiating $\partial u_{k} / \partial \mathbf{n}$ with respect to s. Namely, we have

$$
\frac{\partial}{\partial s}\left(\frac{\partial u_{k}}{\partial \mathbf{n}} \circ \boldsymbol{\gamma}_{k}\right)=\left\|\boldsymbol{\gamma}_{k}^{\prime}\right\| \frac{\partial^{2} u_{k}}{\partial \mathbf{n} \partial \mathbf{t}} \circ \boldsymbol{\gamma}_{k}+\left\langle\nabla u_{k} \circ \boldsymbol{\gamma}_{k}, \frac{\partial \mathbf{n}}{\partial s}\right\rangle
$$

In view of $\partial \mathbf{n} / \partial s=\kappa\left\|\boldsymbol{\gamma}_{k}^{\prime}\right\| \mathbf{t}$ and the homogeneous Dirichlet boundary condition at $\Gamma_{k}$, equation (2.19) implies that

$$
\frac{\partial^{2} u_{k}}{\partial \mathbf{n} \partial \mathbf{t}}=\frac{\partial}{\partial \mathbf{t}}\left(\frac{\partial u_{k}}{\partial \mathbf{n}}\right)-\kappa \frac{\partial u_{k}}{\partial \mathbf{t}}=\frac{\partial}{\partial \mathbf{t}}\left(\frac{\partial u_{k}}{\partial \mathbf{n}}\right) .
$$

According to our smoothness assumptions, the terms $\partial^{2} u_{k} / \partial \mathbf{n}^{2}$ and $\partial^{2} u_{k} / \partial \mathbf{t}^{2}$ are coupled via the Laplace equation, i.e.,

$$
\Delta u_{k}=\frac{\partial^{2} u_{k}}{\partial \mathbf{n}^{2}}+\frac{\partial^{2} u_{k}}{\partial \mathbf{t}^{2}}=0
$$

Hence, the second order directional derivative $\partial^{2} u_{k} / \partial \mathbf{n}^{2}$ can be found from the second order derivative of $u_{k}$ with respect to $s$ :

$$
\begin{aligned}
\frac{\partial^{2}\left(u_{k} \circ \boldsymbol{\gamma}_{k}\right)}{\partial s^{2}} & =\left\langle\left(\nabla^{2} u_{k} \circ \boldsymbol{\gamma}_{k}\right) \cdot \boldsymbol{\gamma}_{k}^{\prime}, \boldsymbol{\gamma}_{k}^{\prime}\right\rangle+\left\langle\nabla u_{k} \circ \boldsymbol{\gamma}_{k}, \boldsymbol{\gamma}_{k}^{\prime \prime}\right\rangle \\
& =\left\|\boldsymbol{\gamma}_{k}^{\prime}\right\|^{2}\left(\frac{\partial^{2} u_{k}}{\partial \mathbf{t}^{2}} \circ \boldsymbol{\gamma}_{k}\right)+\left\langle\boldsymbol{\gamma}_{k}^{\prime \prime}, \mathbf{t}\right\rangle\left(\frac{\partial u_{k}}{\partial \mathbf{t}} \circ \boldsymbol{\gamma}_{k}\right)+\left\langle\boldsymbol{\gamma}_{k}^{\prime \prime}, \mathbf{n}\right\rangle\left(\frac{\partial u_{k}}{\partial \mathbf{n}} \circ \boldsymbol{\gamma}_{k}\right) .
\end{aligned}
$$

This means that

$$
\frac{\partial^{2} u_{k}}{\partial \mathbf{n}^{2}} \circ \boldsymbol{\gamma}_{k}=-\frac{1}{\left\|\boldsymbol{\gamma}_{k}^{\prime}\right\|^{2}} \frac{\partial^{2}\left(u_{k} \circ \boldsymbol{\gamma}_{k}\right)}{\partial s^{2}}+\frac{\left\langle\boldsymbol{\gamma}_{k}^{\prime \prime}, \mathbf{t}\right\rangle}{\left\|\boldsymbol{\gamma}_{k}^{\prime}\right\|^{2}}\left(\frac{\partial u_{k}}{\partial \mathbf{t}} \circ \boldsymbol{\gamma}_{k}\right)-\kappa\left(\frac{\partial u_{k}}{\partial \mathbf{n}} \circ \boldsymbol{\gamma}_{k}\right)
$$

Due to the homogeneous Dirichlet boundary condition at $\Gamma_{k}$, the tangential derivative of $u_{k}$ and the second oder derivative of $u_{k}$ with respect to $s$ disappear. This yields

$$
\frac{\partial^{2} u_{k}}{\partial \mathbf{n}^{2}}=-\kappa \frac{\partial u_{k}}{\partial \mathbf{n}}
$$

The desired equation (2.17) is now an immediate consequence after inserting the equations (2.20) and (2.21) into (2.18).

\section{Discretization}

\subsection{Approximation of the free boundary}

For the numerical computations, we discretize the radial function $r_{k}^{n}$, associated with the boundary $\Gamma_{k}$, by a finite Fourier series according to

$$
r_{k}^{n}(s)=a_{0}+\sum_{i=1}^{n-1}\left\{a_{i} \cos (i s)+b_{i} \sin (i s)\right\}+a_{n} \cos (n s) .
$$

This obviously ensures that $r_{k}^{n}$ is always an element of $C_{\text {per }}^{2}([0,2 \pi])$. To determine the update function $\delta r_{k}^{n}$, represented likewise by a finite Fourier series, we insert the $m \geq 2 n$ equidistantly distributed points $s_{i}=2 \pi i / m$ into the update equation

$$
F\left(\delta r_{k}\right):=\left[\kappa\left(\frac{\partial u_{k}}{\partial \mathbf{n}} \circ \boldsymbol{\gamma}_{k}\right)\left\langle\mathbf{e}_{r}, \mathbf{n}\right\rangle-\frac{\partial}{\partial \mathbf{t}}\left(\frac{\partial u_{k}}{\partial \mathbf{n}} \circ \boldsymbol{\gamma}_{k}\right)\left\langle\mathbf{e}_{r}, \mathbf{t}\right\rangle\right] \delta r_{k}-\frac{\partial u_{k}}{\partial \mathbf{n}} \circ \boldsymbol{\gamma}_{k} \stackrel{!}{=} \lambda .
$$

To solve this equation, we reformulate it as a discrete least-squares problem which can simply be solved via the normal equations. 


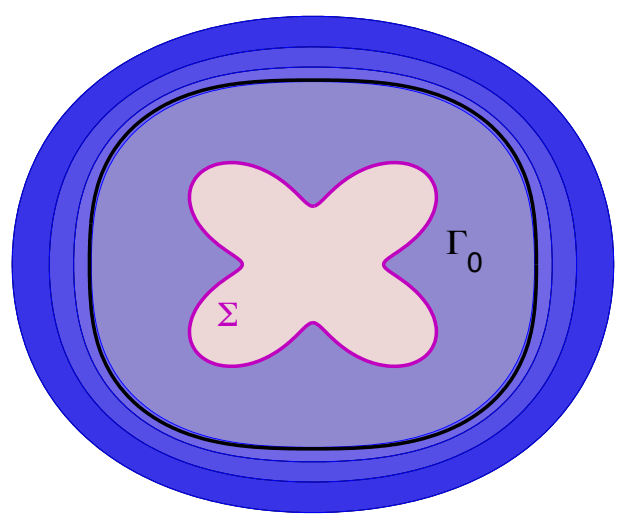

Figure 3.1. Solutions of Bernoulli's free boundary problem in case of an x-shaped interior boundary.

\subsection{Solving the state equation}

The Neumann data of the function $u$ at the actual boundary $\Gamma$ are required for the determination of the update function $\delta r$ and, thus, for the construction of the update rule 1.3. When the solution of a partial differential equation is desired only on the boundary of the domain, then the boundary element method is known to be a very efficient tool. In particular, it enjoys the property of reducing the dimension of the problem by one.

In the present context, the boundary element method has to be applied to the boundary value problem (1.2). To formulate the respective boundary integral equation, we introduce the single layer operator $\mathcal{V}$ and the double layer operator $\mathcal{K}$ defined by

$$
(\mathcal{V} \rho)(\mathbf{x})=-\frac{1}{2 \pi} \int_{\Sigma \cup \Gamma_{k}} \log \|\mathbf{x}-\mathbf{y}\| \rho(\mathbf{y}) \mathrm{d} \sigma_{\mathrm{y}}, \quad(\mathcal{K} \rho)(\mathbf{x})=\frac{1}{2 \pi} \int_{\Sigma \cup \Gamma_{k}} \frac{\left\langle\mathbf{x}-\mathbf{y}, \mathbf{n}_{\mathrm{y}}\right\rangle}{\|\mathbf{x}-\mathbf{y}\|^{2}} \rho(\mathbf{y}) \mathrm{d} \sigma_{\mathrm{y}}
$$

with the densities $\rho$ being the Cauchy data on the boundary $\Sigma \cup \Gamma_{k}$ of the domain $\Omega_{k}$. In accordance with [17, 21], the unknown normal derivative of the state $u_{k}$ is then given by the Dirichlet-to-Neumann map:

$$
\mathcal{V} \frac{\partial u_{k}}{\partial \mathbf{n}}=\left(\frac{1}{2}+\mathcal{K}\right) u_{k} \quad \text { on } \Sigma \cup \Gamma_{k}
$$

First, by using the parametrization of the boundaries as it was described in Assumption 1.1, we parametrize the integral equation (3.4), too. For the approximation of the Cauchy data, we use the collocation method based on trigonometric polynomials on a uniform subdivision of the parameter interval $[0,2 \pi]$. Together with a trapezoidal quadrature rule, this collocation method is converging exponentially under the conditions that the boundaries $\Sigma$ and $\Gamma_{k}$ are smooth and that the solution $u_{k}$ is an analytic function. For more details concerning this boundary element method, in particular, the technique to deal with singular integrals, we refer to [13].

\subsection{Numerical results}

We close this section by presenting some numerical results of the trial method when the update rule (1.3) is used. For this example, we have chosen an ellipse as the initial guess of the free boundary $\Gamma_{0}$ with parametrization

$$
\boldsymbol{\gamma}_{0}:[0,2 \pi] \rightarrow \Gamma_{0}, \quad s \mapsto \boldsymbol{\gamma}_{0}(s)=\sqrt{0.1(\cos (2 s))^{2}+0.12(\sin (2 s))^{2}}\left[\begin{array}{c}
\cos (s) \\
\sin (s)
\end{array}\right]
$$

and an $\mathrm{x}$-shaped fixed boundary $\Sigma$ with parametrization

$$
\boldsymbol{\gamma}_{\Sigma}:[0,2 \pi] \rightarrow \Sigma, \quad s \mapsto \boldsymbol{\gamma}_{\Sigma}(s)=\sqrt{0.01(\cos (2 s))^{2}+0.05(\sin (2 s))^{2}}\left[\begin{array}{c}
\cos (s) \\
\sin (s)
\end{array}\right]
$$

see Figure 3.1 for an illustration.

We intend to solve the overdetermined boundary value problem (1.1) for different values of the constant $\lambda$. The free boundary is represented by 16 degrees of freedom (i.e. $n=8$ in (3.1)) and the Dirichlet-to-Neumann map (3.4) is solved with 600 boundary elements per boundary. The stopping criterion is $\|\delta \mathrm{r}\|<10^{-8}$.

The optimum boundaries $\Gamma^{\star}$ resulting for the different values of $\lambda$ under consideration are also found in Figure 3.1. The associated numbers of iterations of the trial method needed to converge are tabulated in Table 3.1. The results show that convergence of the trial method is achieved only if a damping parameter $(\beta=0.1)$ is used in the update rule (1.3), see the row entitled "update with damping". The damping parameter helps to avoid oscillations of the free boundary during the iteration. However, there is no systematic rule for determining an appropriate damping parameter. Notice that the update rule without damping is not working for any of the chosen parameters $\lambda$ (row entitled "standard update"). 
Table 3.1. Number of iterations of the trial method in case of an x-shaped interior boundary.

\begin{tabular}{ccccccc}
\hline parameter $\lambda$ & 3 & 4 & 5 & 6 & 7 & 8 \\
\hline standard update & - & - & - & - & - & - \\
update with damping $(\beta=0.1)$ & 82 & 73 & 62 & 54 & - & - \\
\hline
\end{tabular}

\section{Convergence analysis}

\subsection{Banach's fixed point theorem}

We shall elaborate the convergence of the trial method by following the technique introduced in [24]. Since the trial method corresponds to the fixed point iteration

$$
r_{k+1}=\Phi\left(r_{k}\right):=r_{k}+\delta r\left(r_{k}\right), \quad k=0,1,2, \ldots,
$$

we can apply Banach's fixed point theorem to the self-mapping $\Phi: X \rightarrow X$, where $X$ stands for the Banach space $C_{\text {per }}^{2}([0,2 \pi])$. The update function $\delta r$ results from equation (2.17). It is given by

$$
\delta r(r)=\frac{\lambda+\frac{\partial u}{\partial \mathbf{n}} \circ \boldsymbol{\gamma}}{\kappa\left(\frac{\partial u}{\partial \mathbf{n}} \circ \boldsymbol{\gamma}\right)\left\langle\mathbf{e}_{r}, \mathbf{n}\right\rangle-\frac{1}{\left\|\boldsymbol{\gamma}^{\prime}\right\|} \frac{\partial}{\partial s}\left(\frac{\partial u}{\partial \mathbf{n}} \circ \boldsymbol{\gamma}\right)\left\langle\mathbf{e}_{r}, \mathbf{t}\right\rangle}
$$

provided that the denominator has no zeros in $[0,2 \pi]$. By construction, this update function $\delta r$ vanishes at the optimum free boundary $\Gamma^{\star}=\left\{\mathbf{x} \in \mathbb{R}^{2}: \mathbf{x}=r^{\star} \mathbf{e}_{r}\right\}$. Hence, the optimum radial function $r^{\star}$ is a fixed point $r^{\star}=\Phi\left(r^{\star}\right)$ of the mapping $\Phi$.

According to Banach's fixed point theorem, there exists a unique solution of the fixed point iteration (4.1) if the mapping $\Phi$ is contractive. The convergence rate

$$
\lim _{k \rightarrow \infty} \frac{\left\|r_{k+1}-r^{\star}\right\|_{X}}{\left\|r_{k}-r^{\star}\right\|_{x}}=\lim _{k \rightarrow \infty} \frac{\left\|\Phi\left(r_{k}\right)-\Phi\left(r^{\star}\right)\right\|_{X}}{\left\|r_{k}-r^{\star}\right\|_{x}}=\lim _{k \rightarrow \infty} \frac{\left\|\delta \Phi\left[r_{k}-r^{\star}\right]\left(r^{\star}\right)\right\|_{X}}{\left\|r_{k}-r^{\star}\right\|_{x}}
$$

can be estimated by

$$
\lim _{k \rightarrow \infty} \frac{\left\|r_{k+1}-r^{\star}\right\|_{x}}{\left\|r_{k}-r^{\star}\right\|_{x}} \leq \sup _{\|q\|_{X}=1} \lim _{\varepsilon \rightarrow 0} \frac{\left\|\Phi\left(r^{\star}+\varepsilon q\right)-\Phi\left(r^{\star}\right)\right\|_{x}}{\varepsilon}=\sup _{\|q\|_{X}=1}\left\|\delta \Phi[q]\left(r^{\star}\right)\right\|_{x} .
$$

Hence, the self-mapping mapping $\Phi$ is (locally) contractive if $\sup _{\|q\|_{x}=1}\left\|\delta \Phi[q]\left(r^{\star}\right)\right\|_{x}<1$.

Theorem 4.1 Consider the trial method based on the update equation (3.2). Then, for a given perturbation $q \in X$, it holds

$$
\delta \Phi[q]\left(r^{\star}\right)=-\frac{1}{\kappa \lambda\left\langle\mathbf{e}_{r}, \mathbf{n}\right\rangle}\left(\frac{\partial \delta u^{\star}[q]}{\partial \mathbf{n}} \circ \boldsymbol{\gamma}^{\star}\right),
$$

where $\boldsymbol{\gamma}^{\star}=r^{\star} \mathbf{e}_{r}$ and $\partial \delta u^{\star}[q] / \partial \mathbf{n}$ denotes the Neumann data of the local shape derivative $\delta u^{\star}[q]$ into the direction $\mathbf{V}=q \mathbf{e}_{r}$.

Proof. Define $\boldsymbol{\gamma}_{\varepsilon}^{\star}=\left(r^{\star}+\varepsilon q\right) \mathbf{e}_{r}$ and let $u^{\star}$ and $u_{\varepsilon}^{\star}$ denote the solutions to the underlying boundary problems (1.2) relative to the domains $\Omega^{\star}$ and $\Omega_{\varepsilon}^{\star}$, i.e.,

$$
\begin{aligned}
\Delta u^{\star}=0 & \text { in } \Omega^{\star}, & \Delta u_{\varepsilon}^{\star}=0 & \text { in } \Omega_{\varepsilon}^{\star} \\
u^{\star}=1 & \text { on } \Sigma, & u_{\varepsilon}^{\star}=1 & \text { on } \Sigma \\
u^{\star}=0 & \text { on } \Gamma^{\star}, & u_{\varepsilon}^{\star}=0 & \text { on } \Gamma_{\varepsilon}^{\star} .
\end{aligned}
$$

On the optimum boundary $\Gamma^{\star}$, the following identities are valid:

$$
u^{\star} \circ \boldsymbol{\gamma}^{\star}=0, \quad-\frac{\partial u^{\star}}{\partial \mathbf{n}} \circ \boldsymbol{\gamma}^{\star}=\lambda \quad \text { and } \quad \delta r\left(r^{\star}\right)=0 .
$$

Hence, we obtain

$$
\begin{aligned}
\delta \Phi[q]\left(r^{\star}\right) & =\lim _{\varepsilon \rightarrow 0} \frac{\Phi\left(r^{\star}+\varepsilon q\right)-\Phi\left(r^{\star}\right)}{\varepsilon} \\
& =\lim _{\varepsilon \rightarrow 0} \frac{r^{\star}+\varepsilon q+\delta r\left(r^{\star}+\varepsilon q\right)-r^{\star}-\delta r\left(r^{\star}\right)}{\varepsilon} \\
& =q+\lim _{\varepsilon \rightarrow 0} \frac{\delta r\left(r^{\star}+\varepsilon q\right)}{\varepsilon}
\end{aligned}
$$


with

$$
\delta r\left(r^{\star}+\varepsilon q\right)=\frac{\lambda+\frac{\partial u_{\varepsilon}^{\star}}{\partial \mathbf{n}_{\varepsilon}} \circ \boldsymbol{\gamma}_{\varepsilon}^{\star}}{\kappa\left(\frac{\partial u_{\varepsilon}^{\star}}{\partial \mathbf{n}_{\varepsilon}} \circ \boldsymbol{\gamma}_{\varepsilon}^{\star}\right)\left\langle\mathbf{e}_{r}, \mathbf{n}_{\varepsilon}\right\rangle-\frac{1}{\left\|\boldsymbol{\gamma}_{\varepsilon}^{\star \prime}\right\|} \frac{\partial}{\partial s}\left(\frac{\partial u_{\varepsilon}^{\star}}{\partial \mathbf{n}_{\varepsilon}} \circ \boldsymbol{\gamma}_{\varepsilon}^{\star}\right)\left\langle\mathbf{e}_{r}, \mathbf{t}_{\varepsilon}\right\rangle}
$$

We split the enumerator of (4.5) as follows

$$
\begin{aligned}
\frac{\partial u_{\varepsilon}^{\star}}{\partial \mathbf{n}_{\varepsilon}} \circ \boldsymbol{\gamma}_{\varepsilon}^{\star}+\lambda & =\frac{\partial u_{\varepsilon}^{\star}}{\partial \mathbf{n}_{\varepsilon}} \circ \boldsymbol{\gamma}_{\varepsilon}^{\star}-\frac{\partial u^{\star}}{\partial \mathbf{n}} \circ \boldsymbol{\gamma}^{\star} \\
& =\left\langle\nabla u_{\varepsilon}^{\star} \circ \boldsymbol{\gamma}_{\varepsilon}^{\star}, \mathbf{n}_{\varepsilon}\right\rangle-\left\langle\nabla u_{\varepsilon}^{\star} \circ \boldsymbol{\gamma}^{\star}, \mathbf{n}_{\varepsilon}\right\rangle+\left\langle\nabla u_{\varepsilon}^{\star} \circ \boldsymbol{\gamma}^{\star}, \mathbf{n}_{\varepsilon}\right\rangle-\left\langle\nabla u_{\varepsilon}^{\star} \circ \boldsymbol{\gamma}^{\star}, \mathbf{n}\right\rangle+\left\langle\nabla u_{\varepsilon}^{\star} \circ \boldsymbol{\gamma}^{\star}, \mathbf{n}\right\rangle-\left\langle\nabla u^{\star} \circ \boldsymbol{\gamma}^{\star}, \mathbf{n}\right\rangle .
\end{aligned}
$$

This yields

$$
\frac{1}{\varepsilon}\left(\frac{\partial u_{\varepsilon}^{\star}}{\partial \mathbf{n}_{\varepsilon}} \circ \boldsymbol{\gamma}_{\varepsilon}^{\star}+\lambda\right) \stackrel{\varepsilon \rightarrow 0}{\longrightarrow} \frac{\partial}{\partial\left(q \mathbf{e}_{r}\right)}\left(\frac{\partial u^{\star}}{\partial \mathbf{n}} \circ \boldsymbol{\gamma}^{\star}\right)+\left\langle\nabla u^{\star} \circ \boldsymbol{\gamma}^{\star}, \frac{\partial \mathbf{n}}{\partial\left(q \mathbf{e}_{r}\right)}\right\rangle+\frac{\partial \delta u^{\star}[q]}{\partial \mathbf{n}} \circ \boldsymbol{\gamma}^{\star} .
$$

Herein, in view of equation (2.14), the derivative of the Neumann data of $u^{\star}$ in the direction $\mathbf{V}=q \mathbf{e}_{r}$ is given by

$$
\frac{\partial}{\partial\left(q \mathbf{e}_{r}\right)}\left(\frac{\partial u^{\star}}{\partial \mathbf{n}} \circ \boldsymbol{\gamma}^{\star}\right)=\left[\left(\frac{\partial^{2} u^{\star}}{\partial \mathbf{n}^{2}} \circ \boldsymbol{\gamma}^{\star}\right)\left\langle\mathbf{e}_{r}, \mathbf{n}\right\rangle+\left(\frac{\partial^{2} u^{\star}}{\partial \mathbf{n} \partial \mathbf{t}} \circ \boldsymbol{\gamma}^{\star}\right)\left\langle\mathbf{e}_{r}, \mathbf{t}\right\rangle\right] q
$$

The Neumann data of the local shape derivative $\delta u^{\star}[q]$ at the boundary $\Gamma^{\star}$ are also contained in (4.6). The local shape derivative satisfies the boundary value problem (2.4). Moreover, the derivative of the normal vector in the direction $\mathbf{V}=q \mathbf{e}_{r}$ fulfills

$$
\frac{\partial \mathbf{n}}{\partial\left(q \mathbf{e}_{r}\right)}=q \frac{\left\langle\mathbf{e}_{r}, \mathbf{t}\right\rangle}{\left\|\boldsymbol{\gamma}^{\star \prime}\right\|} \mathbf{t}-q^{\prime} \frac{\left\langle\mathbf{e}_{r}, \mathbf{n}\right\rangle}{\left\|\boldsymbol{\gamma}^{\star \prime}\right\|} \mathbf{t}
$$

(details about (4.7) are reported in Lemma 2.5). Since the derivative (4.7) of the normal vector is pointing only to the tangential direction, the third term on the right hand side of (4.6) vanishes due to $u^{\star \prime} s$ homogeneous Dirichlet data at $\Gamma^{\star}$ and we arrive at

$$
\lim _{\varepsilon \rightarrow 0} \frac{1}{\varepsilon}\left(\frac{\partial u_{\varepsilon}^{\star}}{\partial \mathbf{n}_{\varepsilon}} \circ \boldsymbol{\gamma}_{\varepsilon}^{\star}+\lambda\right)=\frac{\partial \delta u^{\star}[q]}{\partial \mathbf{n}} \circ \boldsymbol{\gamma}^{\star}+\left[\left(\frac{\partial^{2} u^{\star}}{\partial \mathbf{n}^{2}} \circ \boldsymbol{\gamma}^{\star}\right)\left\langle\mathbf{e}_{r}, \mathbf{n}\right\rangle+\left(\frac{\partial^{2} u^{\star}}{\partial \mathbf{n} \partial \mathbf{t}} \circ \boldsymbol{\gamma}^{\star}\right)\left\langle\mathbf{e}_{r}, \mathbf{t}\right\rangle\right] q .
$$

Finally, we obtain (4.4) by inserting (4.8) into (4.5) and taking into account (2.21) and $-\partial u^{\star} / \partial \mathbf{n} \circ \boldsymbol{\gamma}^{\star}=\lambda$.

On the one hand, the convergence of the trial method is ensured when the mapping $\Phi$ is contractive. This is the case if the norm of (4.4) at $\Gamma^{\star}$ is smaller than 1 , i.e., if

$$
\left\|\delta \Phi[q]\left(r^{\star}\right)\right\|_{X}=\left\|\frac{1}{\kappa \lambda\left\langle\mathbf{e}_{r}, \mathbf{n}\right\rangle}\left(\frac{\partial \delta u^{\star}[q]}{\partial \mathbf{n}} \circ \boldsymbol{\gamma}^{\star}\right)\right\|_{X}<1 .
$$

On the other hand, since for nontrivial boundary perturbations the prescribed Dirichlet data at $\Gamma^{\star}$ in the boundary value problem (2.4) are non-zero, we conclude $\delta u^{\star}[q] \neq 0$. Therefore, in case of convergence, we can only expect a linear convergence order of the trial method.

A much more important consequence of Theorem 4.1 is that the sign of the denominator in (4.4) can change, i.e., the denominator might have zeros and the trial method might thus not converge. Since $\lambda$ is a positive constant and $\left\langle\mathbf{e}_{r}, \mathbf{n}\right\rangle$ is always positive for starlike domains, this happens if the curvature $\kappa$ changes its sign. Hence, the trial method is expected to converge only when the optimum boundary $\Gamma^{\star}$ is convex since the curvature is then positive in a neighbourhood. This observation provides a satisfactory explanation for the results in Section 3.3, where we found that the trial method is converging for those values of $\lambda$ where the optimum boundary is convex.

\subsection{Modified update rule}

Our suggestion to overcome this difficulty is to modify the update rule. Namely, instead of (4.2), we propose to use

$$
\Delta r(r):=\kappa(r) \delta r(r)=\frac{\lambda+\frac{\partial u}{\partial \mathbf{n}} \circ \boldsymbol{\gamma}}{\left(\frac{\partial u}{\partial \mathbf{n}} \circ \boldsymbol{\gamma}\right)\left\langle\mathbf{e}_{r}, \mathbf{n}\right\rangle-\frac{\left\langle\mathbf{e}_{r}, \mathbf{t}\right\rangle}{\kappa\left\|\boldsymbol{\gamma}^{\prime}\right\|} \frac{\partial}{\partial s}\left(\frac{\partial u}{\partial \mathbf{n}} \circ \boldsymbol{\gamma}\right)} .
$$

The self-mapping $\Phi$ is thus modified in accordance with

$$
\Phi_{m}: X \rightarrow X, \quad r \mapsto \Phi_{m}(r)=r+\Delta r(r)
$$


As in Subsection 4.1, we compute the derivative of $\Phi_{m}$ at the point $r^{\star}$ in the direction $q \mathbf{e}_{r}$

$$
\delta \Phi_{m}[q]\left(r^{\star}\right)=\lim _{\varepsilon \rightarrow 0} \frac{\Phi_{m}\left(r^{\star}+\varepsilon q\right)-\Phi_{m}\left(r^{\star}\right)}{\varepsilon}=q+\lim _{\varepsilon \rightarrow 0} \frac{\Delta r\left(r^{\star}+\varepsilon q\right)}{\varepsilon}
$$

and find, likewise to Theorem 4.1, that

$$
\delta \Phi_{m}[q]\left(r^{\star}\right)=q-\frac{1}{\lambda\left\langle\mathbf{e}_{r}, \mathbf{n}\right\rangle}\left(\frac{\partial \delta u^{\star}[q]}{\partial \mathbf{n}} \circ \boldsymbol{\gamma}^{\star}+\kappa \lambda q\left\langle\mathbf{e}_{r}, \mathbf{n}\right\rangle\right)=(1-\kappa) q-\frac{1}{\lambda\left\langle\mathbf{e}_{r}, \mathbf{n}\right\rangle}\left(\frac{\partial \delta u^{\star}[q]}{\partial \mathbf{n}} \circ \boldsymbol{\gamma}^{\star}\right)
$$

For the modified self-mapping $\Phi_{m}$, we obtain thus a denominator which is always positive in a neighbourhood of the optimum boundary $\Gamma^{\star}$, as $\lambda>0$ and $\left\langle\mathbf{e}_{r}, \mathbf{n}\right\rangle>0$ holds in case of starlike domains. Therefore, with the modified update function (4.9), we can expect convergence not only in case of convex optimum boundaries but also in general.

\subsection{Speeding up the convergence}

A further modification of the self-mapping $\Phi$ is obtained by the ansatz

$$
\Phi_{i}: X \rightarrow X, \quad r \mapsto \Phi_{i}(r)=r+\beta(r) \Delta r(r)
$$

with $\Delta r(r)$ from (4.9). The function $\beta(r):[0,2 \pi] \rightarrow \mathbb{R}$ should improve the trial method in two regards. Firstly, it should avoid the necessity to conduct endless tests of the trial method until an appropriate damping parameter is found. Secondly, the function $\beta(r)$ should be chosen such that the speed of convergence is increased.

Since $\Delta r\left(r^{\star}\right)=0$ by construction, the derivative of $\Phi_{i}$ at the point $r^{\star}$ in the direction $q \mathbf{e}_{r}$ is given by

$$
\delta \Phi_{i}[q]\left(r^{\star}\right)=q+\beta\left(r^{\star}\right) \lim _{\varepsilon \rightarrow 0} \frac{\Delta r\left(r^{\star}+\varepsilon q\right)}{\varepsilon} .
$$

In view of (4.10) and (4.11), this derivative reads as

$$
\delta \Phi_{i}[q]\left(r^{\star}\right)=q-\beta\left(r^{\star}\right)\left[\frac{1}{\lambda\left\langle\mathbf{e}_{r}, \mathbf{n}\right\rangle}\left(\frac{\partial \delta u^{\star}[q]}{\partial \mathbf{n}} \circ \boldsymbol{\gamma}^{\star}\right)+\kappa q\right]
$$

The above goals are achieved if we define the function $\beta(r)$ such that the derivative from (4.13) vanishes for the direction $q:=\lim _{k \rightarrow \infty}\left(r_{k}-r^{\star}\right) /\left\|r_{k}-r^{\star}\right\|_{x}$ provided that this limit exists. Since, however, $r^{\star}$ is not known beforehand, $q$ would not be accessible even in the case of existence. Nevertheless, the choice $q_{k}=r_{k}-r_{k-1}$ with $q_{0}=1$ is a good approximation. Hence, we should define the function $\beta$ by

$$
\beta\left(r_{k}\right)=\left[\frac{1}{\lambda\left\langle\mathbf{e}_{r}, \mathbf{n}\right\rangle}\left(\frac{\partial \delta u_{k}\left[q_{k}\right]}{\partial \mathbf{n}} \circ \boldsymbol{\gamma}_{k}\right)+\kappa q_{k}\right]^{-1} q_{k} .
$$

Herein, the local shape derivative $\partial \delta u_{k}\left[q_{k}\right] / \partial \mathbf{n}$ can be computed in complete analogy to the Neumann data of $u_{k}$ by using the Dirichlet-to-Neumann map, as it was described in Section 3. Hence, one additional solve of the Dirichlet-to-Neumann map is necessary per iteration step.

Remark 4.2 The function $\beta\left(r_{k}\right)$ from (4.14) is chosen such that $\delta \Phi_{i}\left[q_{k}\right]\left(r^{\star}\right) \rightarrow 0$ as $k \rightarrow \infty$. However, there are other reasonable definitions with this property. A possible simplification which we use in our particular implementation would be to insert the Neumann data of the solution to the boundary value problem

$$
\begin{aligned}
\Delta \delta \widetilde{u} & =0 & & \text { in } \Omega_{k} \\
\delta \widetilde{u} & =0 & & \text { on } \Sigma \\
\delta \widetilde{u} & =\lambda q_{k}\left\langle\mathbf{e}_{r}, \mathbf{n}\right\rangle & & \text { on } \Gamma_{k}
\end{aligned}
$$

instead of the Neumann data of the local shape derivative $\delta u_{k}\left[q_{k}\right]$ into (4.14). As a further alternative, one may also use

$$
\beta\left(r_{k}\right)=\left[\frac{\frac{\partial \delta u_{k}\left[q_{k}\right]}{\partial \mathbf{n}} \circ \boldsymbol{\gamma}_{k}}{\left(\frac{\partial u}{\partial \mathbf{n}} \circ \boldsymbol{\gamma}_{k}\right)\left\langle\mathbf{e}_{r}, \mathbf{n}\right\rangle-\frac{\left\langle\mathbf{e}_{r}, \mathbf{t}\right\rangle}{\kappa\left\|\boldsymbol{\gamma}_{k}^{\prime}\right\|} \frac{\partial}{\partial s}\left(\frac{\partial u}{\partial \mathbf{n}} \circ \boldsymbol{\gamma}_{k}\right)}+\kappa q_{k}\right]^{-1} q_{k} .
$$

Numerical tests do not clearly show the superiority of one of these choices. 


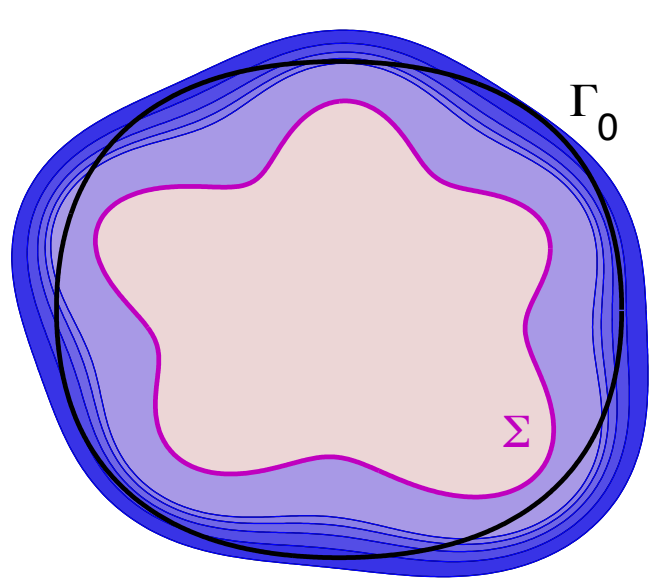

Figure 4.1. Solutions of Bernoulli's free boundary problem in case of a random interior boundary.

Table 4.1. Number of boundary updates of the trial method in case of a random interior boundary.

\begin{tabular}{cccccc}
\hline parameter $\lambda$ & 8 & 10 & 12 & 14 & 16 \\
\hline modified update with damping $(\beta=0.01)$ & 179 & 146 & 130 & 122 & 125 \\
improved modified update & 114 & 94 & 92 & 101 & 92 \\
\hline
\end{tabular}

\subsection{Numerical results}

In our first example, we choose the initial guess $\Gamma_{0}$ of the trial method boundary to be an ellipse parametrized by

$$
\boldsymbol{\gamma}_{0}:[0,2 \pi] \rightarrow \Gamma_{0}, \quad s \mapsto \boldsymbol{\gamma}_{0}(s)=\sqrt{0.1(\cos (2 s))^{2}+0.11(\sin (2 s))^{2}}\left[\begin{array}{c}
\cos (s) \\
\sin (s)
\end{array}\right] .
$$

The fixed interior boundary $\Sigma$ is a randomly generated boundary as seen in Figure 4.1. The solutions of the free boundary problem for different values of $\lambda$ are also found in this figure. For the numerical computations, we have used 50 degrees of freedom for the representation of the free boundary and 600 boundary elements per boundary. The stopping criterion has been $\|\Delta \mathrm{r}\|<10^{-8}$.

In Table 4.1, the number of iterations, which are needed by the trial method to converge, are tabulated. There are no results for the standard update rule (1.3) as in this case the optimum boundary is non-convex for all values of $\lambda$ under consideration, and thus the associated trial method did not converge. In contrast, the trial method based on the modified update rule with update function (4.9) is converging (row entitled "modified update with damping"). Nevertheless, also in this case, a damping parameter $(\beta=0.01)$ is essential for the convergence. By computing the function $\beta(r)$ from (4.14), we avoid not only the costly determination of the damping parameter $\beta$, but we also achieve a slight speed-up of the convergence of the trial method (row entitled "improved modified update").

The second example refers to a domain $\Omega$ which consists of four interior boundaries $\Sigma=\Sigma_{1} \cup \Sigma_{2} \cup \Sigma_{3} \cup \Sigma_{4}$ and one outer free boundaries $\Gamma$. We solve the associated Bernoulli free boundary problem, whose solutions for different values $\lambda$ of the Neumann data are depicted in Figure 4.2. For the numerical simulation have been used: 400 boundary elements per boundary, i.e., 2000 in all, and 30 degrees of freedom for the representation of the free boundary. The initial guess $\Gamma_{0}$ is a properly scaled circle and the stopping criterion is again $\|\Delta r\|<10^{-8}$.

Table 4.2. Number of boundary updates of the trial method in case of several interior boundaries.

\begin{tabular}{ccccccc}
\hline parameter $\lambda$ & 2 & 4 & 6 & 8 & 10 & 12 \\
\hline update with damping $(\beta=0.05)$ & 125 & - & - & - & - & - \\
modified update with damping $(\beta=0.05)$ & 117 & 65 & 49 & 45 & 46 & 51 \\
improved modified update & 118 & 79 & 68 & 48 & 51 & - \\
\hline
\end{tabular}

As it is shown in Table 4.2, we achieve convergence for the trial method and for all the chosen values of the parameter $\lambda$ when the modified update rule (4.9) with damping parameter $\beta=0.05$ is applied. For the same damping parameter, the standard 


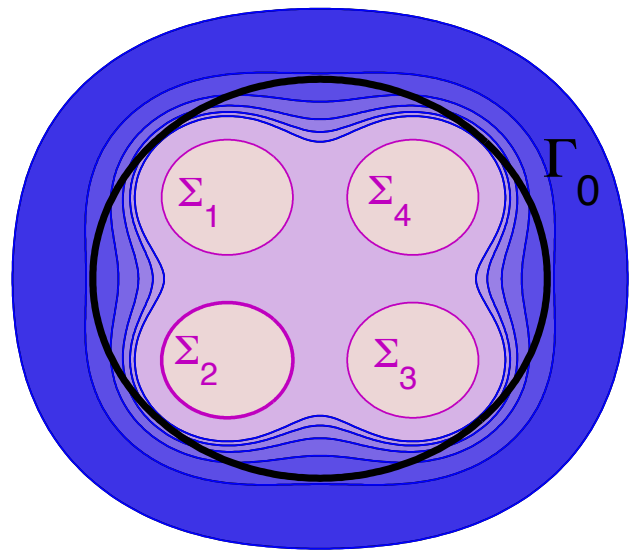

Figure 4.2. Solutions of Bernoulli's free boundary problem in case of several interior boundaries.

update rule (4.2) converges only for $\lambda=2$, as the optimum boundary is convex for this value. The improved modified update rule shows in this case a behaviour which is similar to that of the modified update rule (4.9) with damping parameter.

\section{Conclusion}

In contrast to the trial method which updates according to the Dirichlet data, very few results can be found in the literature about the trial method which updates according to the Neumann data. Here, we elucidated the theoretical background of the latter method and analyzed its convergence. Furthermore, to the best of our knowledge, we are the first who implemented this trial method and presented numerically results in case of more general boundaries and not only axially symmetric ones. For these reasons, we strongly believe that valuable results on this trial method have been achieved in this article, with the most important one being the stabilization of the update equation so that the trial method converges also in case of a nonconvex optimum free boundary.

\section{Acknowledgement}

The authors acknowledge the support of this research by the DFG priority program SPP 1253 Optimization with PDEs and by the SNF through the project No. 200021_137668.

\section{References}

1. A. Acker, Convergence results for an analytical trial free-boundary method, IMA J. Numer. Anal., 8 (1988), pp. 357-364.

2. - On the geometric form of Bernoulli configurations, Math. Methods Appl. Sci., 10 (1988), pp. 1-14.

3. H. W. Alt and L. A. Caffarelli, Existence and regularity for a minimum problem with free boundary, J. Reine Angew. Math., 325 (1981), pp. 105-144.

4. A. Beurling, On free-boundary problems for the Laplace equation, Sem. on analytic functions, Inst. Adv. Stud. Princeton, (1957), pp. 248-263.

5. F. Bouchon, S. Clain, and R. Touzani, Numerical solution of the free boundary Bernoulli problem using a level set formulation, Comput. Methods Appl. Mech. Engrg., 194 (2005), pp. 3934-3948.

6. C. W. Cryer, A survey of trial-boundary methods for the numerical solution of free boundary problems, MRC Techn. Summary Rep., 1693, (1976).

7. M. C. Delfour and J.-P. Zolésio, Shapes and geometries: Metrics, analysis, differential calculus, and optimization, vol. 22 of Advances in Design and Control, Society for Industrial and Applied Mathematics (SIAM), Philadelphia, PA, 2nd ed., 2011.

8. K. Eppler and H. Harbrecht, Tracking Neumann data for stationary free boundary problems, SIAM J. Control Optim., 48 (2009/10), pp. 2901-2916.

9. M. Flucher and M. Rumpf, Bernoulli's free-boundary problem, qualitative theory and numerical approximation, J. Reine Angew. Math., 486 (1997), pp. 165-204.

10. H. Harbrecht and G. Mitrou. Improved trial methods for a class of generalized Bernoulli problems. Preprint 2013-22, Mathematisches Institut, Universität Basel, Switzerland, 2013. 
11. K. Ito, K. Kunisch and G. Peichl, Variational approach to shape derivatives for a class of Bernoulli problems, J. Math. Anal. Appl., 314 (2006), pp. 126-149.

12. K. Kärkkäinen and T. Tiihonen, Free surfaces: shape sensitivity analysis and numerical methods, Internat. J. Numer. Methods Engrg., 44 (1999), pp. 1079-1098.

13. R. Kress, Linear integral equations, vol. 82 of Applied Mathematical Sciences, Springer, New York, 2nd ed., 1999.

14. C. M. Kuster, P. A. Gremaud, and R. Touzani, Fast numerical methods for Bernoulli free boundary problems, SIAM J. Sci. Comput., 29 (2007), pp. 622-634.

15. F. Murat and J. Simon, Etude de probléme d'optimal design, in Proceedings of the 7th IFIP Conference on Optimization Techniques: Modeling and Optimization in the Service of Man, Part 2, Springer, Berlin-Heidelberg-New York, 1976, pp. 54-62.

16. O. Pironneau, Optimal shape design for elliptic systems, Springer series in computational physics, Springer, New York, 1984.

17. S. Sauter and C. Schwab, Boundary Element Methods, Springer, Berlin-Heidelberg, 2010.

18. P. Serranho, A hybrid method for inverse obstacle scattering problems, PhD thesis, Georg-August-Universität Göttingen, 2007.

19. J. Sokolowski and J.-P. Zolésio, Introduction to shape optimization: Shape sensitivity analysis, vol. 16 of Springer Series in Computational Mathematics, Springer, Berlin, 1992.

20. R. V. Southwell and G. Vaisey, Relaxation methods applied to engineering problems. XII. Fluid motions characterized by 'free' streamlines, Philos. Trans. Roy. Soc. London. Ser. A., 240 (1946), pp. 117-161.

21. O. Steinbach, Numerical Approximation Methods for Elliptic Boundary Value Problems. Finite and Boundary Elements, Springer, New York, 2008.

22. D. E. Tepper, Free boundary problem, SIAM J. Math. Anal., 5 (1974), 841-846.

23. T. Tiihonen, Shape optimization and trial methods for free boundary problems, RAIRO Modél. Math. Anal. Numér., 31 (1997), pp. 805-825.

24. T. Tiihonen and J. Järvinen, On fixed point (trial) methods for free boundary problems, in Free boundary problems in continuum mechanics (Novosibirsk, 1991), vol. 106 of Internat. Ser. Numer. Math., Birkhäuser, Basel, 1992, pp. 339-350. 


\section{LATEST PREPRINTS}

No. Author: Title

2013-01 H. Harbrecht, M. Peters

Comparison of Fast Boundary Element Methods on Parametric Surfaces

2013-02 V. Bosser, A. Surroca

Elliptic Logarithms, Diophantine Approximation and the Birch and

Swinnerton-Dyer Conjecture

2013-03 A. Surroca Ortiz

Unpublished Talk: On Some Conjectures on the Mordell-Weil and the Tate-

ShafarevichGgroups of an Abelian Variety

2013-04 V. Bosser, A. Surroca

Upper Bound for the Height of S-Integral Points on Elliptic Curves

2013-05 Jérémy Blanc, Jean-Philippe Furter, Pierre-Marie Poloni

Extension of Automorphisms of Rational Smooth Affine Curves

2013-06 Rupert L. Frank, Enno Lenzmann, Luis Silvestre

Uniqueness of Radial Solutions for the Fractional Laplacian

2013-07 Michael Griebel, Helmut Harbrecht

On the convergence of the combination technique

2013-08 Gianluca Crippa, Carlotta Donadello, Laura V. Spinolo

Initial-Boundary Value Problems for Continuity Equations with BV

Coefficients

2013-09 Gianluca Crippa, Carlotta Donadello, Laura V. Spinolo

A Note on the Initial-Boundary Value Problem for Continuity Equations with Rough Coefficients

2013-10 Gianluca Crippa

Ordinary Differential Equations and Singular Integrals

2013-11 G. Crippa, M. C. Lopes Filho, E. Miot, H. J. Nussenzveig Lopes

Flows of Vector Fields with Point Singularities and the Vortex-Wave System

2013-12 L. Graff, J. Fender, H. Harbrecht, M. Zimmermann

Key Parameters in High-Dimensional Systems with Uncertainty

2013-13 Jérémy Blanc, Immanuel Stampfli

Automorphisms of the Plane Preserving a Curve

Preprints are available under http://math.unibas.ch/research/publications/ 


\section{LATEST PREPRINTS}

No. Author: Title

2013-14 Jérémy Blanc, Jung Kyu Canci

Moduli Spaces of Quadratic Rational Maps with a Marked Periodic Point of Small Order

2013-15 Marcus J. Grote, Johannes Huber, Drosos Kourounis, Olaf Schenk Inexact Interior-Point Method for Pde-Constrained Nonlinear Optimization

2013-16 Helmut Harbrecht, Florian Loos

Optimization of Current Carrying Multicables

2013-17 Daniel Alm, Helmut Harbrecht, Ulf Krämer

The $H^{2}$-Wavelet Method

2013-18 Helmut Harbrecht, Michael Peters, Markus Siebenmorgen

Multilevel Accelerated Quadrature for PDEs with Log-Normal Distributed Random Coefficient*

2013-19 Jérémy Blanc, Serge Cantat

Dynamical Degrees of Birational Transformations of Projective Surfaces

2013-20 Jérémy Blanc, Frédéric Mangolte

Cremona Groups of Real Surfaces

2013-21 Jérémy Blanc, Igor Dolgachev

Automorphisms of Cluster Algebras of Rank 2

2013-22 Helmut Harbrecht, Giannoula Mitrou

Improved Trial Methods for a Class of Generalized Bernoulli Problems

2013-23 Helmut Harbrecht, Johannes Tausch

On Shape Optimization with Parabolic State Equation

2013-24 Zoé Chatzidakis, Dragos Ghioca, David Masser, Guillaume Maurin

Unlikely, Likely and Impossible Intersections without Algebraic Groups

2013-25 Assyr Abdulle, Marcus J. Grote, Christian Stohrer

Finite Element Heterogeneous Multiscale Method for the Wave Equations :

Long Time Effects

2013-26 Luigi Ambrosio and Gianluca Crippa

Continuity Equations and ODE Flows with Non-Smooth Velocity

2013-27 Andriy Regeta

Lie Subalgebras of Vector Fields and the Jacobian Conjecture

2013-28 Helmut Harbrecht, Michael Peters, Markus Siebenmorgen

Tractability of the Quasi-Monte Carlo Quadrature with Halton Points for Elliptic Pdes with Random Diffusion 


\section{LATEST PREPRINTS}

No. Author: Title

2013-29 Helmut Harbrecht, Giannoula Mitrou

Stabilization of the Trial Method for the Bernoulli Problem in Case of Prescribed Dirichlet Data

Preprints are available under http://www.math.unibas.ch/preprints 